\title{
Rendimientos de maíz y soja en sistemas de cultivos en franjas y monocultura: efectos de la orientación de la siembra
}

\author{
Leguizamón, E.S. y D.V. Verdelli
}

\begin{abstract}
RESUMEN
La asociación de cultivos de verano puede ser una alternativa de intensificación en los sistemas productivos de la región pampeana. En un experimento conducido en Monte Buey (Argentina) se evaluó el rendimiento de los sistemas en franjas de maíz-soja, comparados con sus respectivas monoculturas; en el mismo experimento, se evaluó también si diferentes orientaciones de siembra impactan en el rendimiento de ambos sistemas. La intercepción de radiación fotosintéticamente activa (IRFA) en las franjas de maíz sembradas en dirección este-oeste fue mayor y condujo a mayor rendimiento en grano. Las franjas orientadas norte-sur, noreste-suroeste y noroeste-sureste, tuvieron mayor rendimiento que la monocultura, aunque no se evidenció claramente mayor IRFA. En los tratamientos de monocultura de maíz el efecto de la orientación de siembra no produjo cambios en la IRFA y tampoco en el rendimiento. El cultivo de soja en franjas tuvo menor IRFA y menor rendimiento que los tratamientos con monocultura, independientemente de la orientación de siembra. En todos los casos, la asociación de cultivos tuvo valores de eficiencia en el uso de la tierra mayores a 1. El índice de agresividad en todos los casos fue mayor a 0, lo que indica que el maíz tuvo mayor habilidad competitiva que la soja.
\end{abstract}

Palabras clave: cultivo en franja, monocultura, índice de agresividad.

Leguizamón, E.S. and D.V. Verdelli, 2011. Yields of corn and soybean in crops systems in strips and monoculture: effects of the orientation of the seed. Agriscientia XXVIII (2): 147-156

\section{SUMMARY}

The association of summer crops can be an alternative to intensification of production systems of the Pampas. In an experiment conducted in Monte Buey (Argentina), we evaluated the performance of systems in corn-soybean strips, compared with their respective monocultures; in the same experiment were also evaluated whether different planting guidelines impact the performance of both systems. The interception of photosynthetically active radiation (IPAR) in 
the strips of corn planted in an east-west direction was greater and led to higher grain yield. The stripes oriented north-south, northeast-southwest and northwest-southeast, had higher yields than the monoculture; however, the IPAR was not clearly higher. In the corn monoculture treatments the effect of seed orientation did not change neither the IPAR or the crop yield. Soybean cultivation in strips was less IPAR and lower performance than monoculture treatments, regardless of the orientation of planting. In all cases, the association of crops had values of efficiency in land use greater than 1 . The aggressivity index in all cases was greater than 0 , indicating that maize had more competitive ability than soybeans.

Key words: Strip intercropping, monoculture, aggressivity index.

E.S. Leguizamón. Departamento de Sistemas de Producción Vegetal, Facultad de Ciencias Agrarias, Universidad Nacional de Rosario, Zavalla C.C. 14 (SAA2125), Santa Fe, Argentina. D.V. Verdelli. Tesista doctoral, Facultad de Ciencias Agrarias, Universidad Nacional de Rosario, Zavalla. Correspondencia a D. V. Verdelli: dmverdelli@nodosud.com.ar

\section{INTRODUCCION}

La asociación de cultivos consiste esencialmente en el cultivo simultáneo o desfasado de dos o más especies en una misma superficie, con la finalidad de optimizar el uso de los factores y los recursos ambientales (Kantor, 1999). El cultivo en franjas es una de las asociaciones posibles y consiste en la siembra de dos o más especies en franjas "lo suficientemente anchas para permitir las operaciones de siembra y cosecha en forma separada pero lo suficientemente angostas como para permitir la interacción entre ellos" (Andrews \& Kassam, 1976).

En este último aspecto, una planta puede afectar a sus vecinas por cambios en el ambiente. Tales cambios pueden suceder a través de relaciones directas de remoción o adición. Sin embargo, también pueden producirse efectos indirectos sobre el ambiente que afectan a las plantas vecinas, como por ejemplo, cambios en las condiciones de temperatura, radiación, velocidad del viento o alteraciones en el balance de insectos dañinos y benéficos (Altieri, 1992; Finckh \& Mundt, 1992; Sarandon y Chamorro, 2004).

Desde el punto de vista teórico, los recursos disponibles se utilizarían más eficientemente, como resultado de las diferencias en la habilidad competitiva de los componentes de la mezcla (Reddy et al., 1989; Kantor, 1999). El cultivo en franjas es un caso de intersiembra y exhibe los mismos beneficios (Willey, 1990; Keating \& Carberry, 1993; Morris \& Garrity, 1993). Para maximizar los rendimientos en estos sistemas, deben combinarse cultivos y/o épocas de siembra que permitan minimizar o eventualmente evitar por completo el solapamiento de sus periodos críticos (Sarandón y Chamorro, 2004); éste es el caso de franjas de maíz y de soja (Caviglia et al., 2004).

Desde el punto de vista del uso de la radiación, un solo cultivo al año utiliza una pequeña proporción del total disponible; cálculos para cultivos extensivos en la región pampeana, indican que tanto maíz como soja pueden capturar entre 50 y $60 \%$ de la radiación fotosintéticamente activa (RFA) durante la estación de crecimiento (Abbate et al., 1995; Valentinuz, 1996; Della-Maggiora et al., 2000). En maíz, en ambientes sin restricciones de agua y nutrientes, el incremento de la densidad, en un límite estrecho, tiene un efecto positivo en la intercepción de radiación fotosintéticamente activa (IRFA) en el momento alrededor de floración, lo que conduce a un mayor número de granos y rendimiento (Andrade et al., 1993, 1999, 2000; Maddonni et al., 2001; Maddonni \& Otegui, 2004; Maddonni et al., 2006, Westgate et al., 1997). En el caso de soja, Mann \& Jaworski (1970) encontraron en EE.UU, que un sombreado de $63 \%$ por ciento causa abscisión en el 50\% de las vainas. Schou et al. (1978) demostraron que las plantas de soja sombreadas durante el crecimiento reproductivo deprimen el rendimiento de grano; similares respuestas obtuvieron Egli \& Yu (1991). Los resultados expuestos son relevantes a la hora de considerar el efecto del sombreado en las plantas de soja que se encuentran en los bordes de un cultivo con mayor altura, como es el caso del maíz; en este sentido, West \& Griffith (1992) reportan un aumento de $26 \%$ en la producción de maíz pero una reducción de $27 \%$ en 
el rendimiento en la franja de soja contigua al maíz. En el mismo sentido, Ghaffarzadeh et al. (1994) determinaron aumentos de rendimientos de maíz en franjas del orden del $22 \%$ y disminuciones de $12 \%$ en los de soja acompañantes. Más recientemente, Monzón et al. (2005), Díaz et al. (2008) y López et al. (2008) han informado respuestas similares en experimentos realizados en el país.

La comunidad de cultivo intercepta luz solar directa y difusa; las hojas superiores reciben ambos tipos de radiación, mientras que las hojas inferiores reciben una porción más pequeña de radiación directa (Gardner et al., 1985) y a través del canopeo se produce la atenuación de la radiación (Monsi \& Saeki, 2005). Dadas las diferencias en las arquitecturas, los procesos ontogénicos y los determinantes del rendimiento en maíz y en soja, la difusión del sistema de cultivos en franjas depende en gran medida de una mayor generación de conocimientos que contribuyan a explicar las diferencias de rendimientos en las condiciones de la pampa ondulada. En ese sentido, se presume que el estudio de la eficiencia de captación de radiación en relación con la orientación de los surcos es un tema de alta relevancia para contribuir a este propósito.

Este trabajo forma parte de otros experimentos realizados con el objetivo de contribuir a comprender el funcionamiento de los cultivos asociados. Los objetivos de los experimentos contenidos en el presente trabajo consistieron en: (i) comparar el rendimiento de los sistemas de cultivos en monoculturas de maíz y soja, respecto del obtenido en franjas, sembradas según diferentes orientaciones; (ii) evaluar las variaciones de la IRFA en ambos sistemas al modificarse la orientación de la línea de siembra; y (iii) evaluar el grado de intensificación de los sistemas por medio del uso equivalente de la tierra (UET) y el grado de agresividad que cada sistema implica.

\section{MATERIALES Y MÉTODOS}

Los experimentos fueron conducidos en Monte Buey, Prov. de Córdoba, Argentina (32 94' S; $62^{\circ}$ 58' W) durante las campañas 2007-2008 (C1) y 2008-2009 (C2), en un suelo Argiudol Típico, serie Monte Buey (MB1) (Carta de suelos de la República Argentina, 1978), cultivado bajo la modalidad de siembra directa desde 1996.

Los tratamientos consistieron en monoculturas de maíz y de soja, franjas de maíz en el centro de las parcelas, acompañadas en ambos laterales con soja y viceversa, consecuentemente se obtuvieron cuatro formatos de cultivos. Todos ellos se sembraron según cuatro orientaciones: norte-sur (N-S); este-oeste (E-O); noreste-suroeste (NE-SO) y noroeste-sureste (NO-SE), totalizando así 16 tratamientos. El tamaño de la unidad experimental (parcelas) fue de 12 surcos sembrados a 0,52 m entre sí (6,3 m de ancho) por $7 \mathrm{~m}$ de longitud. En los tratamientos que incluyeron las monoculturas, los 12 surcos se sembraron con maíz o soja. En los correspondientes a las franjas, el maíz o la soja se sembraron en la sección central de la parcela (cuatro surcos), además de cuatro surcos a ambos lados, con el cultivo acompañante.

En todos los casos, se utilizó el híbrido de maíz DK 684 RR, híbrido simple de ciclo intermedio (665/685 grados-día desde emergencia a floración femenina y 119 días de madurez relativa) y el cultivar de soja DM 4800RR (grupo de madurez V, con 134/155 grados-día a R8), de hábito de crecimiento indeterminado. Ambos materiales exhibieron buen comportamiento ante enfermedades.

Las siembras de maíz se realizaron el 12/10/07 (C1) y el 14/10/08 (C2), utilizando una densidad de 8,2 plantas $\mathrm{m}^{-2}$. Las siembras de soja se realizaron el 16/11 y el 9/11 en C1 y C2 respectivamente, con una densidad de 24 plantas $\mathrm{m}^{-2}$.

La fertilización nitrogenada en maíz se realizó con UAN (200 kg N ha-1) y la fertilización fosforada con fosfato monoamónico (24 kg N ha ${ }^{-1}$ y 104 kg P ha-1) más una aplicación de yeso (63 kg S ha-1), durante las dos campañas, una semana antes de la siembra. El control de malezas e insectos se realizó según los umbrales de daño establecidos. Para el control de malezas se utilizó glifosato $(74,4 \%)$ a razón de 1,5 L ha-1 de producto comercial en dos momentos de aplicación: antes de la siembra y en estado vegetativo de los cultivos, previo al cierre de surcos; para el control de insectos se realizó una única aplicación, coincidente con la segunda aplicación de herbicidas, con cipermetrina (25\%) a razón de 0,15 L ha-1 de producto comercial. El diseño experimental fue en bloques completos al azar, con cuatro repeticiones. Los datos correspondientes a temperatura, radiación recibida y precipitaciones fueron obtenidos de la estación meteorológica automática situada a 1500 m del sitio experimental.

Durante el crecimiento y el desarrollo del cultivo se determinó la IRFA cada 20 días durante el período siembra-madurez fisiológica. Para ello se utilizó el radiómetro con un sensor lineal de $1 \mathrm{~m}$ de longitud, colocado en diagonal entre los surcos para la medición de la RFA transmitida al suelo y por encima del cultivo para la medición de la radiación RFA recibida por el canopeo. En los tratamientos 
de franjas, las mediciones se efectuaron en ambos bordes del cultivo y en el centro de las parcelas, luego se promediaron sus valores; mientras que en los tratamientos de monocultura, las mediciones se realizaron en la parte central de la parcela y también se promediaron sus valores. En todos los casos las mediciones se efectuaron al mediodía, entre las 11:00 y 14:00 horas. La IRFA por el cultivo se calculó según Gallo et al. (1993):

$$
\text { IRFA }=\text { lo }- \text { Rt } \quad \text { Ecuación } 1
$$

Donde: IRFA es la radiación fotosintéticamente activa interceptada por el cultivo expresada en $\mathrm{Mj}$ $\mathrm{m}^{-2} \mathrm{~d}^{-1}$, lo es la radiación que llega en la parte superior del canopeo y $R t$ la radiación que atraviesa la canopia del cultivo y llega a la superficie del suelo.

El porcentaje de IRFA se calculó a partir de la siguiente ecuación:

$$
\begin{aligned}
& \% I R F A=(I R F A / l o) 100=(1-(R t / / 0)) 100 \\
& \text { Ecuación } 2
\end{aligned}
$$

La RFA recibida durante el ciclo de cultivo, se obtuvo del piranómetro de la estación meteorológi$\mathrm{ca}$, expresada como $\mathrm{Mj} \mathrm{m}^{-2} \mathrm{~d}^{-1}$.

El rendimiento se obtuvo mediante cosecha manual. En todos los tratamientos se cosecharon las plantas de cuatro surcos de $2 \mathrm{~m}$ de longitud, ubicados en el centro de la unidad experimental. Las plantas fueron trilladas, y luego de la limpieza de los granos se registró su peso, corrigiendo la humedad y llevándola a valores de comercialización, 13,5 y $14 \%$ para soja y maíz, respectivamente.

EI UET expresa la superficie de suelo necesaria para lograr el mismo rendimiento en monocultura que en el cultivo en franja. Se calculó con la siguiente ecuación (Vandermeer 1989, 1990):

$$
U E T=\frac{\text { R. Maíz (Franja) + R. Soja (Franja) }}{\text { R. Maíz (Mono) }+ \text { R. Soja (Mono) }}
$$

\section{Ecuación 3}

Donde: R. Maíz (Franja) es el rendimiento promedio de la franja de maíz, R. Soja (Franja) es el rendimiento promedio de la franja de soja, R. Maíz (Mono) es el rendimiento promedio de la monocultura de maíz y R. Soja (Mono) es el rendimiento promedio de la monocultura de soja.

Con los mismos datos también se calculó el índice de agresividad (IA), que mide la competencia interespecífica a través de la relación de los cambios de los rendimientos de los cultivos interactuantes, con la siguiente ecuación (Wiley \& Rao, 1980):

$$
\begin{aligned}
& \text { IA }=\frac{\text { R.Maíz (Franja) }}{\text { R.Maíz (Mono) }{ }^{\star} \text { f. Maíz }}-\frac{\text { R.Soja (Franja) }}{\text { R.Soja (Mono)f S.Soja }} \\
& \text { Ecuación } 4
\end{aligned}
$$

donde: R. Maíz (Franja) es el rendimiento promedio de la franja de maíz, R. Soja (Franja) es el rendimiento promedio de la franja de soja, R. Maíz (Mono) es el rendimiento promedio de la monocultura de maíz, R. Soja (Mono) es el rendimiento promedio de la monocultura de soja y f.Maíz y f.Soja corresponden a las fracciones de superficie ocupadas por maíz y soja, respectivamente.

En todos los tratamientos, las variables fueron analizadas con un ANVA de doble vía (tratamientos y bloques), mediante el software estadístico Statgraphics.

\section{RESULTADOS Y DISCUSIÓN}

\section{Condiciones climáticas}

La RFA recibida por el cultivo en las dos campañas mostró las fluctuaciones características que se suscitan a través del año. Durante el mes de octubre ambos cultivos dispusieron de menor intensidad lumínica que la histórica, porque fueron más frecuentes las condiciones de tiempo nublado y precipitaciones. Hacia la segunda parte del ciclo, desde noviembre hasta fines de enero, cuando el cultivo ya está establecido, la cantidad de radiación solar comenzó a resultar de mayor intensidad. Finalmente, durante el mes de febrero la intensidad lumínica decreció; en ese momento ambos cultivos fenológicamente se encontraron en la etapa de llenado de granos (Fig.1a).

La temperatura media (Fig. 1b) en las dos campañas mostró un incremento desde septiembre a enero, característico de la época, momento a partir del cual comenzó a disminuir. El régimen de precipitaciones fue diferente entre campañas, ya que en C1 se alcanzaron $540 \mathrm{~mm}$ y en C2, $716 \mathrm{~mm}$ en el ciclo de los cultivos, respectivamente (Fig. 1c). En C1 se registró menor cantidad de lluvia caída en el momento inicial del cultivo y también durante el ciclo del cultivo, comparado con C2. Sin embargo, el número de plantas emergidas no fue afectado y en el momento del período crítico de ambos cultivos coincidió con días de precipitación. 

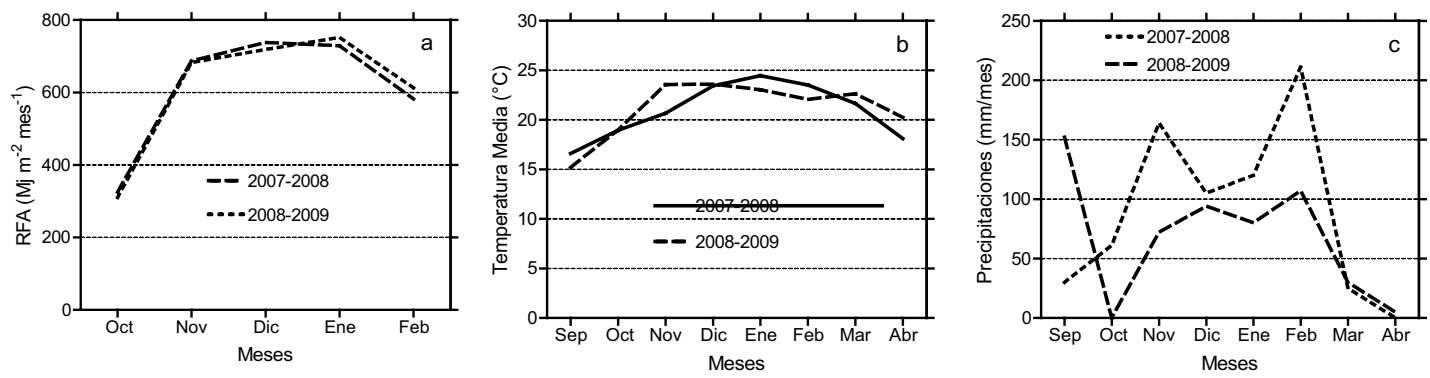

Figura 1. Condiciones meteorológicas: radiación fotosintéticamente activa (RFA) recibida durante el ciclo del cultivo (expresado en Mj $\mathrm{m}^{-2} \mathrm{mes}^{-1}$ ). La RFA recibida en la campaña 2007-08 fue $2614 \mathrm{Mj} \mathrm{m}^{-2}$ en el ciclo de cultivo, $2607 \mathrm{Mj} \mathrm{m}{ }^{-2}$, en la campaña 2008-09, (1a); temperatura media del aire $\left({ }^{\circ} \mathrm{C}\right)(1 \mathrm{~b})$, y precipitaciones mensuales $\left(\mathrm{mm} \mathrm{mes}^{-1}\right)(1 \mathrm{c})$.

Radiación fotosintéticamente activa interceptada por el cultivo (IRFA)

Sin deficiencia de agua y nutrientes, la IRFA alrededor de R1 en el cultivo de maíz, determina su tasa de crecimiento y el número de granos fijados por espiga (Andrade et al., 1996; Andrade et al., 1999). La mayor IRFA en los tratamientos de franjas de maíz, con respecto a la monocultura (Tabla 1), puede deberse a la menor altura de las plantas de soja adyacentes, lo que permitiría al maíz mejorar la captura de este recurso. Esta alta capacidad del maíz en capturar radiación coincide con lo expuesto por West \& Griffith (1992), Fortin \& Pierce (1996), Hauggaard-Nielsen et al. (2001) y Ghosh (2004). Otros estudios revelaron que en asociaciones gramíneas con soja, el sombreamiento que le proporciona el cultivo de mayor porte, afecta la biomasa de la soja debido a la menor asimilación de N, menor síntesis de clorofila y, por consiguiente, una menor tasa fotosintética (Ghosh et al., 2006).

Para el cultivo de maíz en los dos años de evaluación, el tratamiento franja maíz-soja en la orientación E-O tuvo el mayor valor de IRFA durante el ciclo del cultivo, difiriendo significativamente con el resto de los tratamientos y orientaciones, los cua- les no mostraron diferencias entre sí (Tabla 1). Este comportamiento puede atribuirse a que las plantas de los bordes de la franja maíz-soja orientadas E-O tuvieron más tiempo de exposición y penetración de los rayos solares en toda la franja, lo que permitió un mayor valor de IRFA que los tratamientos de monocultura.

En soja, las parcelas con monocultura, en todas sus orientaciones, capturaron mayor radiación que las franjas. La reducción significativa de la IRFA de la franja de soja puede atribuirse al sombreamiento que le proporciona el cultivo de maíz en los bordes de la franja, independientemente de la orientación de siembra. De acuerdo a Hauggaard-Nielsen et al. (2001) y Ghosh (2004), las hileras del cultivo de menor altura, adyacentes al maíz, compiten de manera menos eficiente en la captación de luz. Entre las parcelas de monocultura no se evidenciaron diferencias (Tabla 2).

Por otra parte, el tratamiento de la franja de soja orientada N-S capturó menor IRFA que el resto de las orientaciones de los tratamientos de franja. En este caso, el maíz ejerce un fuerte sombreamiento en los surcos del borde de soja y el tratamiento con orientación N-S sería el más afectado (Tabla 2).

Tabla 1. Valores de IRFA durante el ciclo de cultivo $\left(\mathrm{Mj} \mathrm{m}^{-2}\right.$ ) y su expresión en porcentaje, en el cultivo de maíz para los tratamientos de monocultura y franjas de maíz- soja, con sus diferentes orientaciones de siembra. Letras distintas indican diferencias significativas entre tratamientos $(p<0,05)$.

\begin{tabular}{|c|c|c|c|c|c|c|}
\hline \multirow{3}{*}{ Cultivo } & \multirow{3}{*}{\multicolumn{2}{|c|}{ Tratamiento -orientación }} & \multicolumn{4}{|c|}{ IRFA } \\
\hline & & & \multicolumn{2}{|c|}{ C1 } & \multicolumn{2}{|c|}{$\mathrm{C} 2$} \\
\hline & & & $\mathrm{Mj} \mathrm{m} \mathrm{m}^{-2}$ & $\%$ & $\mathrm{Mj} \mathrm{m}^{-2}$ & $\%$ \\
\hline \multirow{8}{*}{ Maíz } & \multirow{4}{*}{ Franja maíz-soja } & $\mathrm{E}-\mathrm{O}$ & 1147(a) & 44 & 1163(a) & 45 \\
\hline & & N-S & 978(b) & 37 & 991(b) & 38 \\
\hline & & NO-SE & 986(b) & 38 & 999(b) & 38 \\
\hline & & NE-SO & 986(b) & 38 & 999(b) & 38 \\
\hline & \multirow{4}{*}{ Monocultura maíz } & $\mathrm{E}-\mathrm{O}$ & 1009(b) & 39 & 1028(ab) & 39 \\
\hline & & N-S & 990(b) & 38 & 1003(b) & 38 \\
\hline & & NO-SE & $991(b)$ & 38 & 1004(b) & 39 \\
\hline & & NE-SO & 983(b) & 38 & 994(b) & 38 \\
\hline
\end{tabular}


Tabla 2: Valores de IRFA durante el ciclo de cultivo $\left(\mathrm{Mj} \mathrm{m}^{-2}\right)$ y su expresión en porcentaje, en el cultivo de soja para los tratamientos de monocultura y franjas de soja- maíz, con sus diferentes orientaciones de siembra. Letras distintas indican diferencias significativas entre tratamientos $(p<0,05)$.

\begin{tabular}{|c|c|c|c|c|c|c|}
\hline \multirow{3}{*}{ Cultivo } & \multirow{3}{*}{\multicolumn{2}{|c|}{ Tratamiento -Orientación }} & \multicolumn{4}{|c|}{ IRFA } \\
\hline & & & \multicolumn{2}{|c|}{ C1 } & \multicolumn{2}{|c|}{ C2 } \\
\hline & & & $\mathrm{Mj} \mathrm{m}^{-2}$ & $\%$ & $\mathrm{Mj} \mathrm{m} \mathrm{m}^{-2}$ & $\%$ \\
\hline \multirow{8}{*}{ Soja } & \multirow{4}{*}{ Franja soja-maíz } & $\mathrm{E}-\mathrm{O}$ & $880(a)$ & 34 & 891(a) & 34 \\
\hline & & N-S & $765(b)$ & 29 & $776(b)$ & 30 \\
\hline & & NO-SE & $858(a)$ & 33 & 869(a) & 33 \\
\hline & & NE-SO & 849(a) & 32 & $860(a)$ & 33 \\
\hline & \multirow{4}{*}{ Monocultura soja } & $\mathrm{E}-\mathrm{O}$ & 1039(c) & 40 & 1050(c) & 40 \\
\hline & & N-S & $996(c)$ & 38 & $1007(\mathrm{c})$ & 39 \\
\hline & & NO-SE & 997(c) & 38 & $1008(c)$ & 39 \\
\hline & & NE-SO & 984(c) & 38 & 995(c) & 38 \\
\hline
\end{tabular}

\section{Rendimiento}

El crecimiento de los cultivos depende de la radiación solar incidente, de la capacidad del canopeo para interceptarla y de la eficiencia con que el cultivo transforma la radiación interceptada en materia seca (Gardner et al., 1985). Por otra parte, el rendimiento queda determinado por la manera en que el cultivo particiona la biomasa acumulada durante su crecimiento, entre los órganos de cosecha y el resto de la planta (Andrade et al., 1996).

En C1, el cultivo de maíz bajo el sistema de franja orientado E-O, tuvo mayor rendimiento que la monocultura en todas sus orientaciones (Fig. 2a). Sin embargo, el maíz cultivado en franja en las orientaciones restantes (N-S, NE-SO y NO-SE), no se diferenció estadísticamente de las parcelas de monocultura ni de la franja E-O. Por su parte, el rendimiento en los tratamientos con monocultura de maíz no se diferenciaron entre sí en ninguna orientación de siembra (Fig. 2a).

En C2, el rendimiento del maíz bajo el sistema en franjas, orientadas E-O y NO-SE, se diferenció estadísticamente con todas las orientaciones de la monocultura (Fig. 2b). Los rendimientos de las franjas de maíz orientadas NE-SO y N-S se diferenciaron con el de la monocultura orientada $\mathrm{E}-\mathrm{O}$ y NE-SO. El rendimiento del maíz en el sistema de monoculturas no se diferenció entre sí en ninguna de las orientaciones (Fig. 2b).

Según lo expuesto, las franjas tienen mayor rendimiento que las monoculturas, aunque no en todos los casos estas diferencias son significativas estadísticamente, pero existe un consenso generalizado en que las franjas de un cultivo alto tiene mayores y mejores oportunidades para capturar recursos, lo que permite obtener mayores rendimientos que la monocultura (Lesoing \& Francis, 1990; Boehner et al., 1991; Fortin et al., 1994; Abbate et al., 1995). El aumento de rendimiento del maíz en las franjas puede deberse a un aumento en la tasa de crecimiento del cultivo (TCC) lo que conduciría a una mayor acumulación de biomasa, de acuerdo
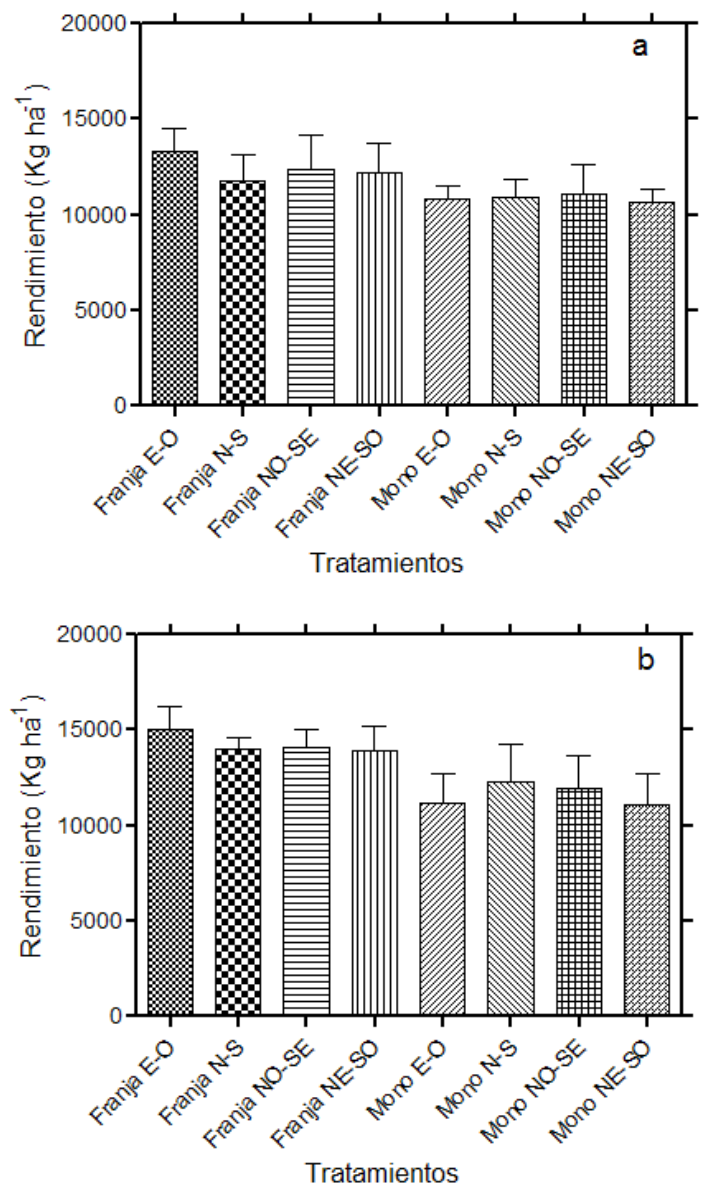

Figura 2. Rendimiento de maíz en los sistemas de franja y monocultura, en diferentes orientaciones de siembra en las campañas 2007-08 (Fig. 2a) y 2008-09 (Fig. 2b). 
a lo reportado por Fortin et al. (1994); Ghaffarzadeh et al. (1994, 1997); Iragavarapu \& Randall (1996); Lesoing \& Francis (1999 a,b).

En C1 y C2, el rendimiento de soja en el sistema de monocultura fue significativamente superior que el de la soja cultivada en franjas, en todas las orientaciones de siembra (Fig. 3 a y b). El rendimiento en la monocultura de soja no mostró diferencias en las distintas orientaciones. En el sistema en franjas, la soja con orientación N-S tuvo menor rendimiento que el resto de los tratamientos en franjas (NO-SE, $\mathrm{E}-\mathrm{O}$ y NE-SO), y estas diferencias fueron significativas. Esto puede ser explicado por la menor cantidad de IRFA recibida en su ciclo (Tabla 1). Varios autores reportaron reducción de rendimiento de las hileras del borde lindantes con el maíz, lo que conduce a un menor rendimiento en toda la parcela (Pendleton et al., 1963; Francis et al., 1978; West \& Griffith, 1992; Ghaffarzadeh et al., 1994; Lesoing
\& Francis, 1999 a,b), debido a la menor posibilidad de capturar recursos. Otros estudios revelaron en los sistemas de cultivos en franjas entre gramíneas-soja, el sombreo efectuado por cultivos altos puede afectar de manera adversa la producción de biomasa, nodulación, disponibilidad de nitrógeno, síntesis de clorofila y por lo tanto la fotosíntesis del cultivo de menor porte (Ghosh et al., 2006).

\section{IRFA y Rendimiento}

En maíz, en todos los casos se observó que un aumento de la IRFA se reflejó en mayor rendimiento, comportamiento que también fue reportado por Andrade et al. (2000). En C1, el rendimiento fue menor comparado con C2, presumiblemente por la menor cantidad de precipitaciones. Por tal motivo, en la Fig.4 la recta de regresión en C1 se desplazó hacia abajo con respecto a C2. Por otra parte, se produjo un incremento de rendimiento en
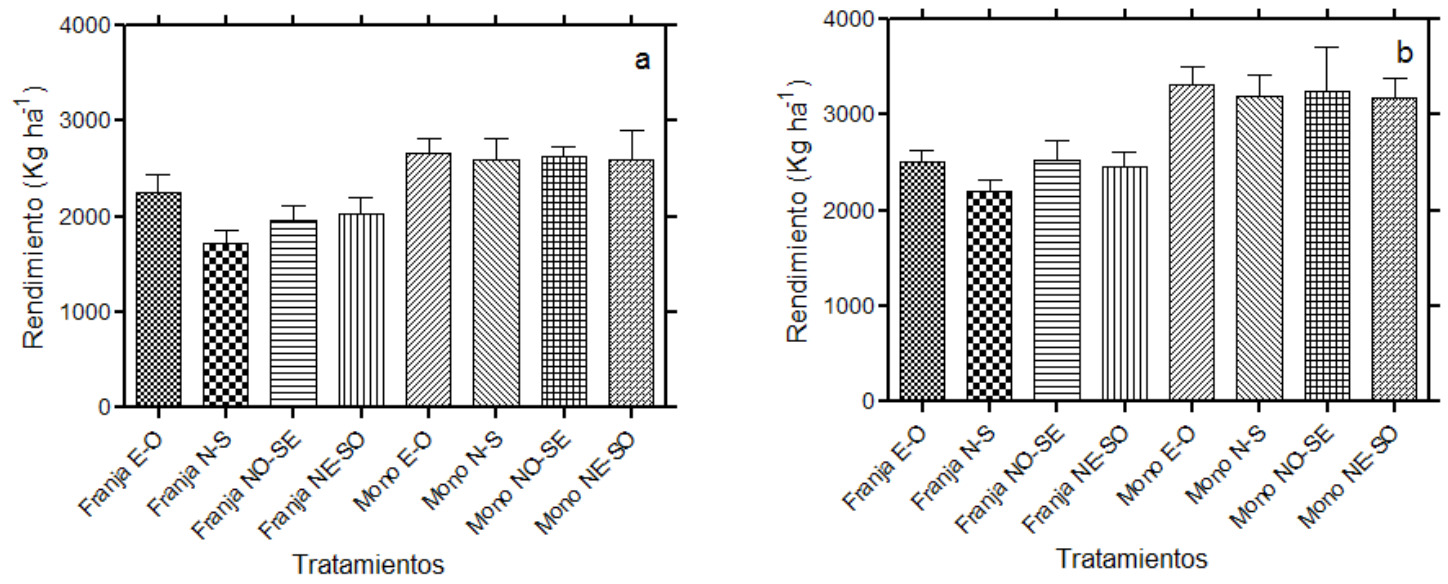

Figura 3. Rendimiento de soja en los sistemas de franja y monocultura, en las diferentes orientaciones de siembra en las campañas 2007-08 (Fig. 1 a) y 2008-09 (Fig. 1 b).
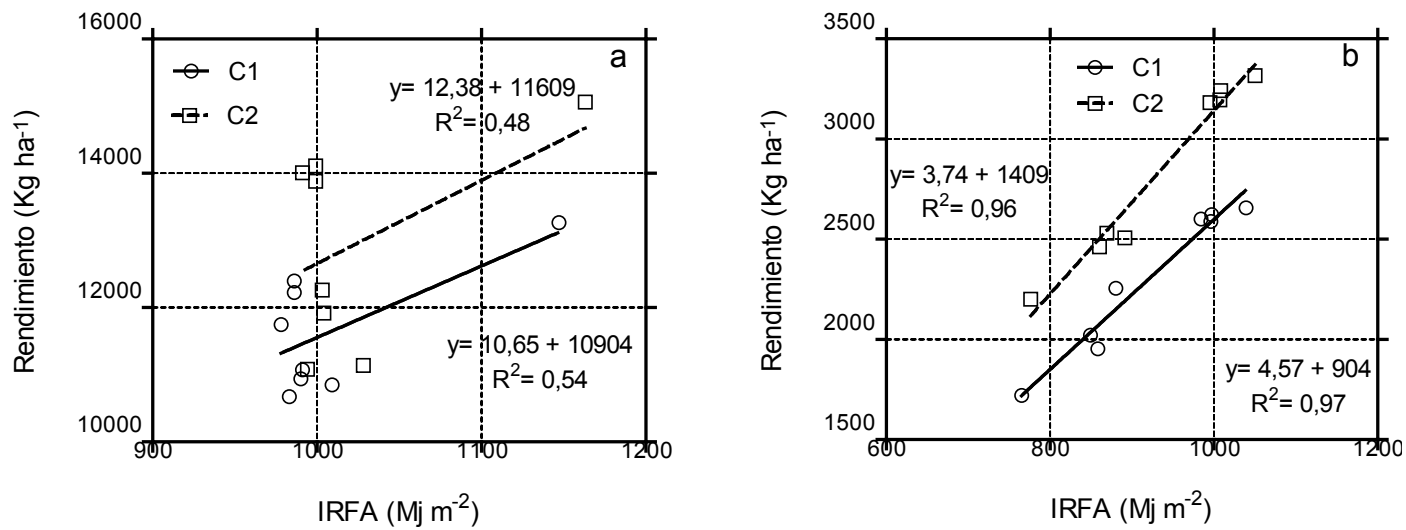

Figura 4. Rendimiento de maíz (a) y soja (b) $\left(\mathrm{kg} \mathrm{ha}^{-1}\right)$ en relación a la intercepción de radiación fotosintéticamente activa recibida durante el ciclo de cultivo (Mj m²) en todos los tratamientos, en las campañas 2007-08 (C1) y 2008-09 (C2). 
grano cuando aumentó la radiación interceptada, (Fig. 4a).

En soja, también se observó que un aumento de la IRFA produjo mayor rendimiento. Del mismo modo que en maíz, en $\mathrm{C} 1$ el rendimiento fue menor comparado con C2 (Fig. 4b). Datos similares fueron reportados por Andrade et al. (2000).

\section{UET y Agresividad}

Desde el punto de vista del sistema, una forma de evaluar los efectos de los cultivos en franjas es mediante el cálculo del uso equivalente de la tierra (UET). Este índice es igual a la suma de las fracciones de rendimiento de los cultivos integrantes del intercultivo en relación con los rendimientos de los cultivos en monocultura; expresa la fracción de tierra necesaria para que con los cultivos individuales se obtengan los valores del intercultivo (Monzón, 2005; Caviglia, 2007). La Tabla 3 muestra los valores de UET en la asociación maíz-soja comparados con la monocultura (UET=1). Los resultados obtenidos muestran que la asociación de cultivos en todos los años tuvo valores mayores a 1, lo que indica mayor eficiencia en el uso del suelo que la monocultura y que existe una tendencia hacia la complementariedad en el uso de los recursos (Vandermeer, 1989; 1990). Sin embargo, sólo las franjas sembradas en las orientaciones E-O y NESO mostraron diferencias significativas en el valor del UET con respecto a la monocultura en las dos campañas. Las orientaciones de siembra N-S y NO-SE sólo se diferenciaron estadísticamente de la monocultura en C2, aunque en las dos campañas el valor de UET fue mayor que 1. Estos valores de EUT son similares a los reportados por Vandermeer (1989, 1990), Willey (1990) y West \& Griffith (1992) (Tabla 3).

El índice de agresividad, en todos los tratamientos de asociación, mostró valores mayores a 0, lo que indica la mayor habilidad competitiva del maíz en la asociación que el cultivo de soja, (Tabla 4). Esto posiblemente se deba a una mayor eficiencia en el uso de los recursos (luz, agua y nutrientes) y

Tabla 3. Valores de UET en los diferentes tratamientos. Valores mayores a 1 indican que la asociación maíz soja utilizó más eficientemente el suelo que la monocultura, como así también el principio de facilitación contribuye en mayor medida que los fenómenos de competencia.

\begin{tabular}{lcc}
\hline Tratamiento & C1 & C2 \\
\hline Franja E-O & $1,2(\mathrm{a})$ & $1,2(\mathrm{a})$ \\
Franja N-S & $1,2(\mathrm{bc})$ & $1,1(\mathrm{~b})$ \\
Franja NO-SE & $1,1(\mathrm{bc})$ & $1,1(\mathrm{~b})$ \\
Franja NE-SO & $1,1(\mathrm{~b})$ & $1,2(\mathrm{a})$ \\
Monocultura & $1,0(\mathrm{c})$ & $1,0(\mathrm{c})$ \\
\hline
\end{tabular}

Tabla 4. Valores de agresividad en la asociación maíz soja.

\begin{tabular}{lcc}
\hline Tratamiento & C1 & C2 \\
\hline Franja E-O & 0,75 & 1,19 \\
Franja N-S & 0,56 & 0,78 \\
Franja NO-SE & 0,75 & 0,81 \\
Franja NE-SO & 0,74 & 0,89 \\
\hline
\end{tabular}

a su capacidad de transformarlo en grano (Andrade et al., 2000). Por otra parte, la soja, además de ser una especie C3, tiene un alto costo energético para producir granos debido a la deposición de aceite en la etapa final del Ilenado.

\section{CONCLUSIONES}

El cultivo de maíz en franjas, orientadas E-O, tuvo mayor IRFA que el resto de las orientaciones, las cuales no mostraron diferencias entre ellas. Sólo la orientación E-O se diferenció estadísticamente del maíz en monocultura.

El cultivo de soja, en todas las orientaciones de siembra (E-O, N-S, NE-SO y NO-SE), bajo el sistema en franjas capturó menor IRFA que todos los tratamientos en monocultura, debido presumiblemente al sombreamiento ejercido por el maíz en la frontera de cultivos. En los tratamientos de monocultura, la orientación de siembra no produjo cambios en la IRFA. El rendimiento de la soja en las franjas fue menor en todas las orientaciones de siembra comparados con la monocultura; la dirección N-S fue la más afectada.

El aumento de IRFA produjo aumentos de rendimiento en maíz y soja en ambos años, pese a que las precipitaciones fueron distintas entre años.

En todos los tratamientos, el UET fue mayor a 1, y el índice de agresividad mayor a 0.

\section{AGRADECIMIENTOS}

Al señor G. Verdelli, que facilitó el predio para la realización de los ensayos; al señor J.R. Perez y a los alumnos de sexto año del Instituto Técnico Agrario Industrial (ITAI), por sus aportes en la toma de muestras.

\section{BIBLIOGRAFIA}

Abbate, P.E.; F.H. Andrade and J.F. Culot, 1995. The effects of radiation and nitrogen on number of grain in wheat. J. Agric. Sci. 124:351-360.

Altieri, M.A., 1992. Diversidad vegetal y estabilidad de poblaciones de insectos en agroecosistemas. En: M.A. Altieri (Ed.) Biodiversidad, agroecología y manejo de 
plagas, Cetal Ediciones, Valparaíso, Chile. pp. 41-53.

Andrade, F.H.; S.A. Uhart and M.I. Frugone, 1993. Intercepted radiation at flowering and kernel number in maize: Shade versus plant density effects. Crop Sci. 33:482-485.

Andrade, F.; A. Cirilo; S. Uhart y M. Otegui, 1996. Ecofisiología del cultivo de maíz. Editoral Médica Panamericana. pp 20.

Andrade, F.H.; C. Vega; S. Uhart; A. Cirilo; M. Cantarero and O. Valentinuz, 1999. Kernel number determination in maize. Crop Sci. 39:453-459.

Andrade, F.H.; L.A.N. Aguirrezábal y R.H. Rizzalli, 2000. Crecimiento y rendimiento comparados. En Bases para el manejo del maíz, el girasol y la soja, Eds., F. H. Andrade and V. O. Sadras. INTA-Universidad de Mar del Plata, Balcarce. pp. 61-96.

Andrade, F.H.; C. Vega; S. Uhart; A. Cirilo; M. Cantarero and O. Valentinuz, 1999. Kernel number determination in maize. Crop Sci. 39:453-459.

Andrews, D.J and A.H. Kassam, 1976. Importance of multiple cropping in increasing world food supplies. pp. 1-10, En: R.I. Papendick, A. Sanchez, and G.B. Triplett (eds.) Multiple Cropping. ASA (American Society of Agronomy) Spec. Pub. 27.

Boehner, P.; G. Lesoing and C.A. Francis, 1991. Strip cropping of grain sorghum/soybeans under dryland and irrigation in eastern Nebraska. In Agronomy abstracts 1991. ASA, Madison, WI. p. 139.

Carta de suelos de la República Argentina, Hoja 3363-17 Marcos Juárez (INTA 1978).

Caviglia O.P., 2007. La contribución de los cultivos múltiples a la sustentabilidad de los sistemas agrícolas en Entre Ríos. Agricultura Sustentable en Entre Ríos. Editores: Caviglia, O.P.; Paparotti, O.F. y Nasal, M.C. Ediciones INTA 2007. pp. 139-148.

Caviglia O.P.; V.O. Sadras and F.H. Andrade, 2004. Intensification of agriculture in the south-eastern Pampas. I. Capture and efficiency in the use of water and radiation in double cropped wheat-soybean. Field Crops Res. 87:117-129.

Della-Maggiora, A.I.; J.M. Gardiol y A.I. Irigoyen, 2000. Requerimientos hídricos. En: Andrade, F.H., Sadras, V.O. (Eds), Bases para el manejo del maíz, girasol y la soja. Ed. Médica Panamericana S.A., Balcarce, pp. 155-171.

Díaz, M.G.; W. Kuttel, R. Lopez, O. Caviglia, H. Peltzer y E. Blanzaco, 2008. Evaluación de diferentes proporciones de maíz-soja en intercultivo en surcos. INTA-EA Paraná FCA (UNER).

Egli, D.B. and Z. Yu, 1991. Crop qrowth rate and seed per unit area in soybean. Crop Sci. 31:439-442.

Finckh, M.R. y C.C. Mundt, 1992. Stripe rust, yield and plant competition in wheat cultivar mixtures. Phytopathology, 82: 905-913.

Fortin M.C.; J. Culley and M. Edwards, 1994. Soil water, plant growth, and yield of strip-intercropped corn. J. Prod. Agric. 7:63-69.

Fortin, M.C. and F.J. Pierce, 1996. Leaf azimuth in stripintercropped corn. Agron. J. 88:6-9

Francis C.A.; C.A. Flor and M. Prager, 1978. Effects of bean association on yields and yield components of maize. Crop Sci. 18:760-764.

Gallo, K. P.; C.S.T. Daughtry and C.L. Wiegand, 1993. Errors in measuring absorbed radiation and computing crop radiation use efficiency. Agron. J. 85:1222-1228.

Gardner, B.R.; R.B. Pearce and R.L. Michell, 1985. Physiology of crops plants. Iowa State University Press. USA.

Ghaffarzadeh M.; F.G. Préchac and R.M. Cruse, 1994. Grain yield response of corn, soybean, and oat grown in a strip intercropping system. Am. J. Altern. Agric. 9:171-177.

Ghaffarzadeh, M.; F.G. Préchac and R.M. Cruse, 1997. Tillage effect on soil water content and corn yield in a strip intercropping system. Agron. J. 89:893-899

Ghosh, P.K.; M.C. Manna, K.K. Bandyopadhyay Ajay, A.K. Tripathi, R.H. Wanjari, K.M. Hati, A.K. Misra, C.L. Acharya and A. Subba Rao, 2006. Interspecific Interaction and Nutrient Use in Soybean/Sorghum Intercropping System. Agron. J. 98 (4):1097-1108.

Ghosh, P.K., 2004. Growth, yield, competition and economics of groundnut/cereal fodder intercropping in the semi-arid tropics of India. Field Crops Res. 88:227-237.

Hauggaard-Nielsen, H.; P. Ambus, and E.S. Jensen, 2001. Temporal and spatial distribution of roots and competition for nitrogen in pea-barley intercrops. A field study employing 32P technique. Plant Soil 236:63-74.

Iragavarapu, T.K. and G.W. Randall, 1996. Border effects on yields in a strip-intercropped soybean, corn, and wheat production system. J. Prod. Agric. 9:101-107

Kantor, S., 1999. Intercropping. Cooperative Extension Washington State University King County. Disponible en: www.king.wsu.edu (consultado el 14/07/10).

Keating, B.A. and P.S. Carberry, 1993. Resource capture and use in intercropping: solar radiation. Field Crops Res. 34:273-301.

Lesoing, G.W. and C.A. Francis, 1990. Strip cropping of corn and soybean under dryland and irrigated conditions. p. 150. In Agronomy abstracts 1990. ASA, Madison, WI.

Lesoing, G. and C. Francis. 1999a. Strip Intercropping Effects on Yield and Yield Components of Corn, Grain 
Sorghum, and Soybean. Agron. J. 91:807-813.

Lesoing, G. and C. Francis, 1999b. Strip intercropping of grain sorghum/soybean in irrigated and rainfed environments. J. Prod. Agric. pp. 601-606.

López, R.; M.G. Díaz; W. Kuttel; O. Caviglia y H. Peltzer, 2008. Evaluación de diferentes proporciones de maízsoja en intercultivo en surco para rendimiento de grano. Análisis combinado de las campañas 2006/07 - 2007/08. INTA-EEA Paraná FCA (UNER).

Maddonni, G.A.; M. Chelle; J.L. Drouet and B. Andrieu, 2001. Light interception of contrasting azimuth canopies under square and rectangular plant spatial distributions: Simulations and crop measurements. Field Crops Res. 70:1-13.

Maddonni, G.A. and M.E. Otegui, 2004. Intra-specific competition in maize: Early establishment of hierarchies among plant affects final kernel set. Field Crops Res. 85:1-13.

Maddonni, G.A.; A.G. Cirilo and M.E. Otegui, 2006. Row width and maize grain yield. Agron. J. 98:1532-1543.

Mann, J.D. and E.J. Jaworski, 1970. Comparison of stresses which may limit soybean yields. Crop. Sci. 10:620-624.

Monsi, M. and T. Saeki. 2005. On the factor light in plant communities and its importance for matter production. Annals of Botany 95: 549-567.

Monzón J.P.; T.J. Carrozzo; O.P. Calviño y F.H. Andrade, 2005. Efecto del intercultivo en franjas de maíz y soja sobre el rendimiento. Actas VII Congreso Nacional de Maíz. 16 al 18 de Noviembre de 2005. Rosario, Argentina. pp. 64-66.

Morris, R.A. and D.P. Garrity, 1993. Resource capture and utilization in intercropping: water. Field Crops Res. 34:303-317.

Pendleton J.W.; C.D. Bolen and R.D. Seif, 1963. Alternating strips of corn and soybeans vs. solid plantings. Agron.

\section{J. 55:293-295.}

Reddy, S.N.; E.V.R. Reddy, V.M. Reddy, M.S. Reddy and P.V. Reddy, 1989. Row arrangement in groundnut/ pigeonpea intercropping. Tropical Agric. 66:309-312.

Sarandon, S.J. y A.M. Chamorro, 2004. Policultivos en los sistemas de producción de granos. En: Satorre E.H. (Ed.), Producción de granos bases funcionales para su manejo. Ed. FAUBA, Bs As., Argentina, pp. 353372.

Schou, J.B.; D.L. Jeffers and J.G. Streeter, 1978. Effects of reflectors, black boards, or shades applied at different stages of plant development on yield of soybean. Crop. Sci. 18:29-34.

Valentinuz, O.R., 1996. Crecimiento y rendimiento comparados de girasol, maíz y soja ante cambios en la densidad de plantas. M.Sc. Thesis. UNMdP, Balcarce.

Vandermeer, J.H., 1989. The ecology of intercropping. Cambridge University Press. Cambridge, New York. 20 pp.

Vandermeer, J.H., 1990. Intercropping. In: Agroecology (eds Carrol, C.R., Vandermeer, J. H. \& Rosset, P. M.). McGraw and Hill, New York, NY, pp.481-516.

West, T.D. and D.R. Griffith, 1992. Effect of strip intercropping corn and soybean on yield and profit. J. Prod. Agric. 5:107-110.

Westgate, M.E.; F. Forcella; D.C. Reicosky and J. Somsen, 1997. Rapid canopy closure for maize production in the northern US corn belt: Radiation-use efficiency and grain yield. Field Crops Res. 49:249-258.

Willey R.W. and M.R. Rao, 1980. A competitive ratio for quantifying competition between intercrops. Exp. Agric. 16:117-125.

Willey, R.W., 1990. Resource use in intercropping systems. Agricultural Water Management. 17:215-23. 